\title{
Expansion Planning for Transmission Network under Demand Uncertainty: \\ A Real Options Framework
}

Fikri Kucuksayacigil ${ }^{\mathrm{a}}$ and K. Jo Min ${ }^{\mathrm{a}^{*}}$

\begin{abstract}
In recent years, there has been much expectation that transmission expansion planning should address ever increasing demands for transmission services under significant and complex economic and regulatory uncertainties. In this paper, towards meeting the aforementioned expectation, we develop and analyze a real options framework that provides the valuation of a transmission owner's option to expand in his/her network. What distinguishes our framework from the extant literature is that the evolution of the demand follows a Geometric Brownian motion process, it explicitly accounts for the physical flow of the electric power economically manifested as locational marginal prices, and it shows how the values of the expansion options can be determined in the transmission network. Furthermore, our framework shows how to value an option to expedite or delay can be determined given that a specific expansion is planned. An extensive numerical example is presented so as to illustrate the key features of our framework.
\end{abstract}

Keyword: Transmission Expansion Planning, Real Options, Locational Marginal Price Running Head: Value of transmission expansion under uncertainty

\footnotetext{
${ }^{a}$ Department of Industrial and Manufacturing Systems Engineering, Iowa State University, Ames, Iowa 50011, USA.

*Corresponding author. Phone: 515-294-8095. Fax: 515-294-3524. E-mail: jomin@iastate.edu
} 


\section{Introduction}

Since the deregulation of the US electric power of 1990's, the transmission aspect of the electric power industry has been separated from the generation aspect, and the responsibilities of the transmission network owners have been much different from the responsibilities of generation unit decision makers (we will use owners and decision makers interchangeably as the decisions made in this paper are on behalf of the owners). For example, many generation unit decision makers have no obligation to serve while transmission owners are expected to address increasing demands and still maintain technical requirements such as reliability and stability. For this reason, there have been numerous sophisticated studies on transmission expansion planning (see e.g., Buygi et al. 2004), which is characterized by uncertainties ranging from demands to fuel costs, substantial and upfront expansion investment costs, and irreversibility of the expansion investment.

In often-practiced case of the hybrid merchant/regulated mechanism for the expansion investment, a major part of the revenue needed for expansion is collected from the market participants such as distribution utilities and power generators. e.g., in California, participating transmission owners, who obey the regulatory authority of the independent system operator, are allowed to collect the Transmission Access Charge (TAC; California 2014a, California 2014b). The other major part is through the Financial Transmission Rights (FTR's), which depends on the Locational Marginal Price (LMP) differences. In this mechanism, the transmission network owners hold FTR's, and sells them to market participants to generate the other major part of the revenue needed for expansion (see e.g., Pringles et al. 2014).

We note that, from the perspective of numerous transmission owners in the hybrid merchant/regulated mechanisms, the expansion (and when to do it given that they would do it) can be viewed as strategic real options offering managerial flexibility under uncertainties (see 
e.g., Dixit and Pindyck 1994).

In this paper, for such owners in the hybrid merchant/regulated mechanisms, we show how the values of the expansion options can be determined in the transmission network. Furthermore, our framework shows how to value an option to expedite or delay can be determined given that a specific expansion is planned. This is achieved under the assumption that the evolution of the demand follows a Geometric Brownian motion process and there are no other uncertainties, and through the optimal power flow calculations leading to the appropriate LMP levels.

The rest of the paper is organized as follows. We first present a review of the relevant literature. Next, we explain the general framework of our paper. This is followed by an extensive numerical example that illustrates the key features of our framework. Finally, we make concluding remarks and provide a technical appendix on LMP calculations.

\section{Literature Review}

For electricity market, the real options approach has frequently been studied for generation expansion planning. As for transmission expansion planning, the real options approach has been less frequently studied. Of such studies on transmission expansion planning, there primarily are three groups of real options applications.

In the first group, the configuration of the transmission network is simply bi-nodal (a network of two nodes). Hence, there is only one investment decision under consideration (i.e., to add a power line between the two nodes; see e.g., Abadie and Chamorro 2011).

The second group of studies investigates the option to defer the transmission investment. In this case, one can separate such studies into two subgroups based on their network configurations. In one subgroup, the researchers quantify the option to defer in bi-nodal networks 
(see e.g., Blanco et al. 2009). In the other subgroup, the researchers quantify the option to defer in multi-node networks of three or more nodes (see e.g., Osthues et al. 2014).

In the third group, there exist studies focusing on special electrical devices such as flexible AC transmission systems (FACTS) and distributed generations. Blanco et al. (2011) evaluates the transmission investment in FACTS devices. In their model, transmission owner either invests in a transmission line or FACTS devices that relieve the transmission congestion. Similarly, Vasquez and Olsina (2007) focuses on the deferral effect of distributed generation in transmission investment. The authors claim that the owner can postpone the investment in transmission network by constructing distributed generation units that relieve the transmission congestion.

\section{General Framework}

In this section, we will first elaborate the revenue being generated by LMP differences, the binomial lattice construction for demand uncertainties. This is followed by the investment valuation process with a flowchart. We note that the optimal power flow (OPF) background to facilitate the derivation of the LMP differences is provided as an appendix.

\section{Revenue Generated by LMP Differences}

When generators are dispatched at optimality and if they are paid according to their own LMPs and the consumption centers pay for electricity according to their own LMPs, then there exists a surplus amount of money (see, e.g., Pe'rez-Arriaga et al. (2013), Hsu (1997)). This surplus results from the congestion in the network and it is generally accepted as the source of revenue for the network owner (see, e.g., Garcia et al. (2010), Pringles et al. (2014)). This revenue is modeled as in Equation (1). 


$$
\sum_{i \in N_{D}} \pi_{i} D_{i}-\sum_{j \in N_{G}} \pi_{j} G_{j}
$$

where $\pi_{i}$ denotes the LMP at node $i, D_{i}$ denotes the demand amount at node $i$ and $G_{j}$ denotes the dispacthed amount of power from generator at node $j$ (at optimality of OPF problem), $\left|N_{D}\right|$ and $\left|N_{G}\right|$ denotes the set of consumption centers and generator nodes, respectively. Krause (2003) states that Equation (1) is always larger than zero if at least one transmission line is congested. If none of the power lines is congested, then the difference equals to zero. Although we cannot present the detail of this payment mechanism, we utilize this difference as the revenue of the network. We note that this revenue is hourly basis (the unit is $\$ / \mathrm{h}$ ) because the unit of LMPs is dollars per MWh.

\section{Uncertainty and Discretization by Binomial Lattice}

Since the option evaluation based on a continuous stochastic process such as geometric Brownian motion process is difficult, we intend to use the discretized form of this process. Before going on the demand growth in multiple consumption centers, we focus on a demand evolution in a single consumption center in order to present the lattice discretization more clearly. After that, we introduce the multiple branch lattices for illustrating the demand growth in multiple consumption centers.

\section{$\underline{\text { Single Consumption Center }}$}

In this section, taking into account the uncertain demand growth in a consumption center, we discuss the derivation of binomial lattice parameters, discount rates and the importance and derivation of risk-neutral probability. We will address more complex multiple consumption centers afterwards. 


\section{Derivation of Parameters for Binomial Lattice}

One of the most commonly used discretization method is binomial lattice developed by Cox et al. (1979). According to this method, a variable $X$ (in our case, $X$ represents $D_{i}$ where $i$ is the single consumption center) has two possibilities in the next period; it either goes up or goes down. The change in $X$ is determined by the multiplication factors $u>1$ and $d<1$. In other words, it becomes either $u X$ or $d X$ with probabilities $p$ and $(1-p)$, respectively (Figure 1). Therefore, the parameters, $u, d$ and $p$ should be found.

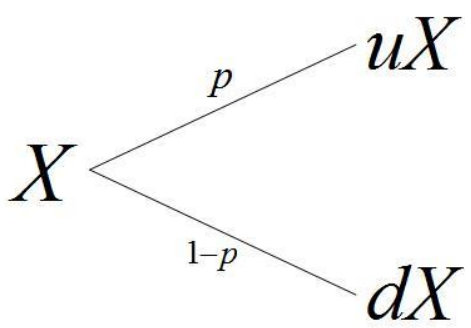

Figure 1. One-step lattice

Discretization of geometric Brownian motion process can be performed by considering the natural logarithm of the change in $X$, which is denoted as $\ln (X)$. Binomial lattice matches the expected value and variance of $\ln (X)$. By following the derivation procedure shown by Luenberger (1997), the parameters can be obtained as follows:

$$
\begin{gathered}
u=e^{\sigma \sqrt{\Delta t}} \\
d=e^{-\sigma \sqrt{\Delta t}} \\
p=\frac{1}{2}+\frac{1}{2}\left(\frac{\mu-\frac{1}{2} \sigma^{2}}{\sigma}\right) \sqrt{\Delta t}
\end{gathered}
$$

where $\sigma$ is the volatility of the process $X$ and $\Delta t$ is the length of the time period in lattice. We remark that the probability $p$ is derived from the discretization of geometric Brownian motion 
process; hence, it is not risk-neutral probability. In binomial lattice calculations, risk-neutral probability should be used rather than $p$.

\section{Discount Rates}

A discount rate here is the interest rate used in discounted cash flow analysis to calculate the present value of the future cash flows. Discount rate takes into account not only time value of the money, but also risk included in future cash flows (see, e.g., Investopedia (2014)).

If it is not desired to include the risk, then it is viable to utilize the risk-free discount rate. Zacks (2014) states that risk-free discount rate is typically the amount that an owner expects to gain at the end of investing in a zero risk security. In general, the yield on a U.S. Government bond is accepted as risk-free discount rate.

In the context of company businesses, different discount rates are used for evaluating the projects because of the fact that projects have risk. According to Investopedia (2014), weighted average cost of capital is generally used in the case that project risk profile is similar to company's risk profile. On the other hand, if they are different, capital asset pricing model is frequently used to determine the project-specific discount rate. Discount rate calculated by this way is called risk-adjusted discount rate.

Risk-adjusted discount rate is defined as the sum of risk-free discount rate and risk premium (see, e.g., Investopedia (2014)). Risk premium can be calculated as the (market rate of return - risk free discount rate) multiplied by beta of the project. More specifically, Investopedia (2014) defines that beta of the project represents the extend "how much a company's share price moves against the market as a whole". If beta is equal to one, then they move in line with each other. Otherwise, if it is larger than one, then the share is said to exaggerate the movements of market, and if it is less than one, then it is said to be more stable. 


\section{$\underline{\text { Risk-Neutral Probability }}$}

In binomial lattice calculations, risk has to be included inside the equations. Mun (2002) states that cash flows including risk must be adjusted so that risk can be represented. According to Mun (2002), there exist two methods for doing this: (i) cash flows are calculated by utilizing the risk-adjusted discount rate or (ii) probabilities of the cash flows are adjusted with risk and discount of cash flows is performed with risk-free discount rate. While original (or true) probabilities are taken into account during calculations in (i), risk-neutral probabilities are considered during calculation in (ii). The methods defined in (ii) is preferred in real options analysis because it is expressed that calculating different risk-adjusted discount rates in various nodes through binomial lattice is avoided in this case. The following simple example depicted in Figure 2 (see, Mun (2002)) explains how risk-neutral probability is obtained.

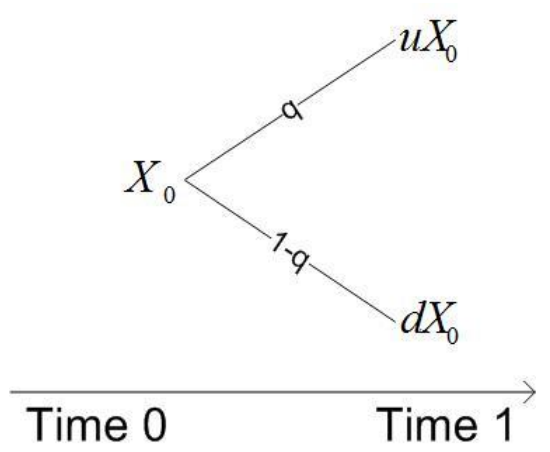

Figure 2. Simple payoff

Let $X_{0}$ be the payoff of a game. The expected payoff at time $0\left(X_{0}\right)$ is simply calculated as

$$
X_{0}=\left(q u X_{0}+(1-q) d X_{0}\right) \cdot(1+r)^{-1}
$$

where $r$ is the risk-free discount rate. By assuming that $X_{0}=1$, then 


$$
1=(q u+(1-q) d) \cdot(1+r)^{-1}
$$

which gives risk-neutral probability (for up movement) as

$$
q=\frac{1+r-d}{u-d}
$$

where $u$ and $d$ are calculated by using Equation (2) and (3). Alternatively, in Wang and Min (2006), it is stated that risk-neutral probability can be found by replacing $\mu$ in Equation (4) with $r$. We now present more general case of multiple consumption centers.

\section{$\underline{\text { Multiple Consumption Centers }}$}

If demand growth is an uncertain factor in multiple nodes, then binomial lattice turns into multiple branch lattice because demand in consumption center $i, D_{i}$, has different drift and volatility parameters than those of demand in consumption center $j, D_{j}$. A state in lattice consists

of the demand vector $\left(D_{1}, D_{2}, \ldots, D_{\left|N_{D}\right|}\right)$ where $N_{D}$ is the set of consumption centers. Hence, the number of branches from one point to the next point is $2^{\left|N_{D}\right|}$. In lattice, to calculate the demands in the next period, all $u_{i}$ and $d_{i}$ possibilities are taken into account in a permutational manner. Figure 3 illustrates an example of demand evolution in two consumption centers for two periods. 


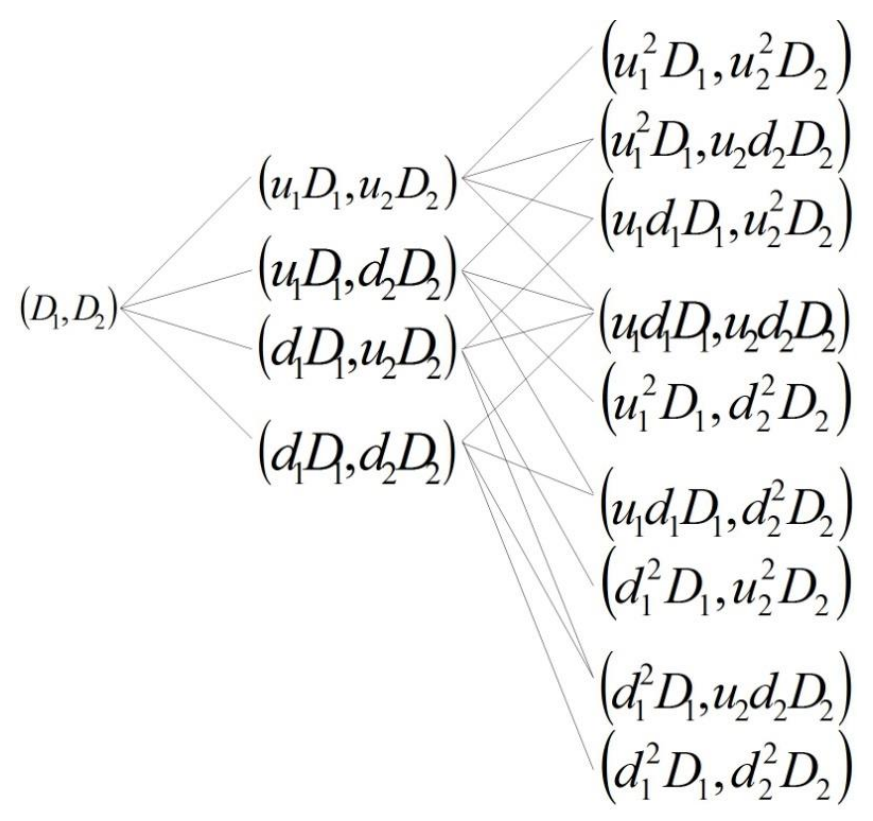

\begin{tabular}{l|l} 
Period 1 & Period 2
\end{tabular}

Figure 3. Multiple branch lattice

Although finding the permutation of all $u_{i}$ and $d_{i}$ at the beginning of next period is easy, it is not easy to come up with the probabilities of the branches in multiple branch lattices similar to Equation (4). According to Wang and Min (2006), if there is no correlation between the demand growths, then the joint probabilities of the branches can be found with the multiplication of marginal probabilities. If there is a correlation between demand growths, then

$$
q_{k}=\prod_{i=1}^{\left|N_{D}\right|} p_{\delta_{i}(k)}+\frac{1}{2^{\left|N_{D}\right|}} \sum_{i=1}^{\left|N_{D}\right|} \sum_{j=i+1}^{\left|N_{D}\right|} \delta_{i j}(k) \rho_{i j}, \quad k=1,2, \ldots\left|N_{D}\right|
$$

gives the joint probability for branch $k$, where $\rho_{i j}$ is the correlation coefficient. Moreover, $\delta_{i}(k)$ and $\delta_{i j}(k)$ are defined as follows:

$$
\delta_{i}(k)= \begin{cases}u_{i}, & \text { if demand in node } i \text { has up movement in branch } k \\ d_{i}, & \text { if demand in node } i \text { has down movement in branch } k\end{cases}
$$




$$
\begin{aligned}
& \delta_{i j}(k) \\
& =\left\{\begin{aligned}
1, & \text { if demand in } i \text { and } j \text { move in the same direction in branch } k \\
-1, & \text { if demand in } i \text { and } j \text { move in the opposite direction in branch } k
\end{aligned}\right.
\end{aligned}
$$

We note that in Equation (8), $p_{u_{i}}$ is the probability defined in Equation (4). Since we need risk-neutral probabilities, we first convert $p_{u_{i}}$ to the risk neutral probability $q_{u_{i}}$ by replacing $\mu_{i}$ in Equation (4) with $r$. Then, if we use $q_{u_{i}}$ instead of $p_{u_{i}}$ in Equation (8), we can obtain riskneutral probability of branch $k$.

Before going into detail of transmission investments evaluation, in Table 1, we present the notations frequently used during creation of the evaluation lattices. As mentioned earlier, we now elaborate on the investment valuation process.

\section{Investment Valuation Process}

To facilitate the understanding of the critical flowchart in this subsection, we first provide the following list of notations.

Table 1

Notations for the Investment Valuation Process

\begin{tabular}{cl}
\hline Notation & \multicolumn{1}{c}{ Explanation } \\
\hline$t$ & A period in multiple branch lattice \\
$T$ & Last period
\end{tabular}


$V_{t k} \quad$ Net present value of the network in NPV lattice at the beginning of period $t$ and state $k(\$)$. Thus, as opposed to $N P V_{t k}$, it includes additionally risk-neutral expected value of the next period. (see Equation (13))

$V_{T k} \quad$ Net present value of the network in NPV lattice at the beginning of period $T$ and state $k(\$)$

$D M C \quad$ Decommissioning cost of the network (\$)

A Supplementary revenue for the owner (\$)

I Initial investment cost $(\$)$

Given the notations in Table 2, the general process for the investment valuation is as follows. For a given network, an investment alternative is defined as adding a power line between selected two nodes. In the valuation approach, the investment alternatives between each pair of nodes are evaluated separately.

The general flowchart for evaluating all investment alternatives existing in the network can be seen in Figure 4.

Box number <1>: In this step, an investment alternative such as adding a power line between node 1 and 2 in the network is selected. The set of investment alternatives are defined as the collection of each investment alternative between a pair of nodes in the network.

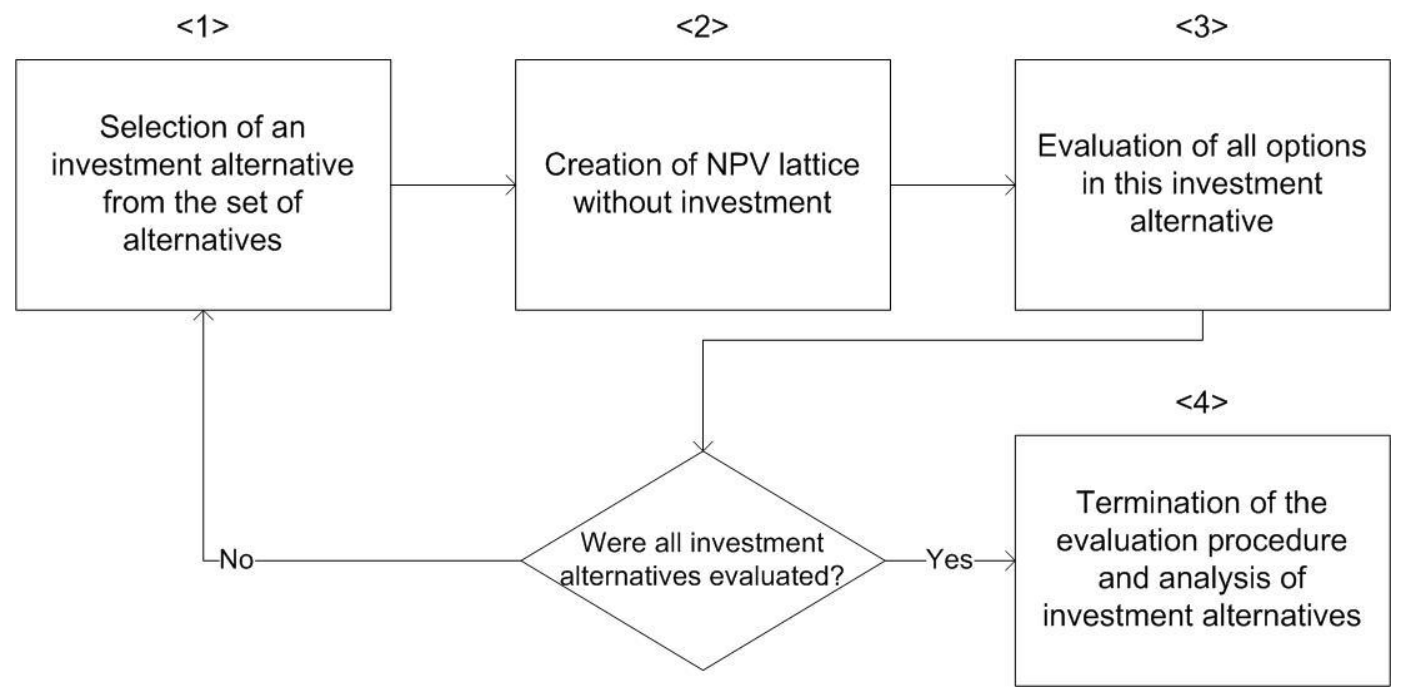

Figure 4. Flowchart for investment alternatives evaluation 
Box number <2>: In this step, NPV lattice for the network without investment is created. Constructing the binomial lattice starts from backward. Thus, terminal node values $\left(V_{T k}\right)$ should be determined, at first. At the terminal nodes, LMP-based revenues and corresponding profits are calculated for one year. For the corresponding demand values at the beginning of period $T$ and state $k$, we calculate the LMPs by solving the OPF problem. Then, by using Equation (1), network revenue denoted by $N R_{t k}$ is computed and $N P V_{t k}$ is calculated by using Equation (11). In addition to $N P V_{t k}$ for the terminal node, we add the discounted decommissioning cost with Equation (12). Thus, we obtain $V_{T k}$ for the terminal node.

$$
\begin{gathered}
N P V_{t k}=H \cdot\left(N R_{t k}-c\right) \cdot(1+r)^{-\Delta t} \\
V_{T k}=N P V_{t k}+(-D M C)^{-\Delta t}
\end{gathered}
$$

For the intermediate node, after calculating the corresponding $N P V_{t k}$ with Equation (11), we add it the risk neutral expected value of the next period successor states (corresponding $V_{t+1, k}$ ) by using Equation (13). Thus, we find the NPV value of the network at present time denoted by $V_{11}$ by the recursive relation presented in Equation (13).

$$
V_{t k}=N P V_{t k}+\left(\sum_{k \in S_{E_{t k}}} q_{k} V_{t+1, k}\right) \cdot(1+r)^{-\Delta t}
$$

Box number <3>: This procedure has a sub-procedure illustrated in Figure 5. Option $t$ represent the investment made at the beginning of the period $t$. Therefore, for a model horizon being equal to $T$, the owner has $T$ options to evaluate. In each option, at the end of period $T$, a decommissioning cost is incurred. Moreover, we assume that transmission access charge $A$ and 
initial investment cost $I$ is incurred whenever an investment is made.

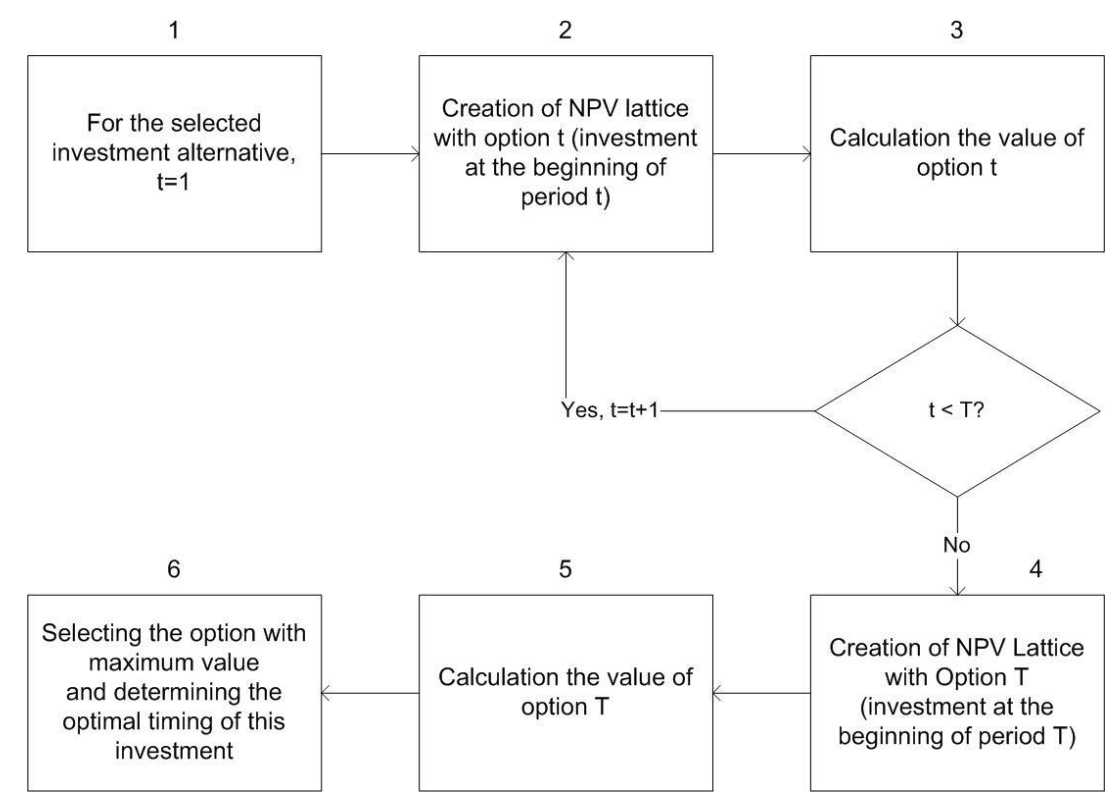

Figure 5. Flowchart of evaluation of options existing in one investment alternative

Box number 2: Option $t$ represents that an investment is made at the beginning of period $t<T$. Creating binomial lattice again starts with the terminal node and proceeds by backward induction. Thus, at the terminal node, we can still use the same equations, Equation (11) and (12). However, we note here that since an investment is made before T, LMPs and LMP-based revenue $\left(N R_{t k}\right)$ calculations are performed with respect to the new network configuration. For an intermediate node after $t$, we can still use the same equation, Equation (13). We again note that during calculating the LMPs and LMP-based revenues, new network configurations should be taken into account. At the beginning of period $t$, since an investment is made at that time point, we should add $A$ and subtract $I$ in Equation (13). For period before $t$, we can still use Equation (13), but we note that since an investment is not available at that time point, LMPs and LMPbased calculations are performed by considering the network configuration without investment. Thus, with the recursive relations, $V_{11}$ is obtained with the investment made at the beginning of 
period $t$.

Box number 3: The value of the option $t$ is simply calculated as the difference between $V_{11}$ of NPV lattice with option $t$ and $V_{11}$ of NPV lattice without investment. If the latter one is larger than the former one, then we say that value of option $t$ is zero.

Box number 4: Option $T$ represents the situation in which an investment is made at the beginning of period $T$. In that case, at the terminal node, the owner still collects the revenue based on LMP differences, represented by Equation (11). Since decommissioning cost is incurred at the end of period $T$, the corresponding cost should still be considered in Equation (12). However, $A$ must be added and I must be subtracted in Equation (12) because the owner makes an investment at the beginning of period $T$. We note that, for option $T$, LMPs and LMPbased revenue calculations are all performed with the upgraded network configuration at the terminal node. For the intermediate nodes, we can still use Equation (13), but network configuration without investment should be taken into account during calculation of LMPs and LMP-based revenues. Thus, this recursive relations, we provide $V_{11}$ value at the present time with option $T$.

Box number 5: There does not exist any difference between methods in box number 3 and box number 5 . In other words, the value of option $T$ is calculated as the difference between $V_{11}$ of NPV lattice with option $T$ and $V_{11}$ of NPV lattice without investment. If the former one is less than the latter one, then the value of option $T$ is said to be zero.

Box number 6: In this step, values of all options are evaluated. Since it is better to have larger value, option with the maximum value is preferred. It also reveals the optimal timing of the investment.

Now, we turn to the upper procedure depicted in Figure 4. In box number <4>, 
investment alternatives are compared according to their optimal timing and their values.

\section{Numerical Example}

In this section, a small but comprehensive numerical example on three-node network is presented. As can be seen in Figure 6, there are two generators at node 1 and 2. The capacity of the first generator $\left(\overline{G_{1}}\right)$ is $100 \mathrm{MW}$ and its generation cost $\left(C_{1}\right)$ is $\$ 40 / \mathrm{MWh}$. The second generator capacity $\left(\overline{G_{2}}\right)$ is $200 \mathrm{MW}$ and its generation cost $\left(C_{2}\right)$ is $\$ 30 / \mathrm{MWh}$. We note that supply curves of these generators are assumed to be linear and not to change for the sake of simplification (see, e.g., California ISO (2005)). In other words, generator 1 is willing to produce each additional unit of electricity at $\$ 40 / \mathrm{MWh}$ up to $100 \mathrm{MW}$ and generator 2 is willing to produce each additional unit of electricity at \$30/MWh up to $200 \mathrm{MW}$.

The capacity of the power lines $\left(\overline{L_{12}}, \overline{L_{13}}, \overline{L_{23}}\right)$ are $30 \mathrm{MW}, 36 \mathrm{MW}$ and $35 \mathrm{MW}$, respectively. There is a consumption center at node 3 and the load amount $\left(D_{3}\right)$ is 52 MW. Susceptance of the power lines are assumed to be equal.

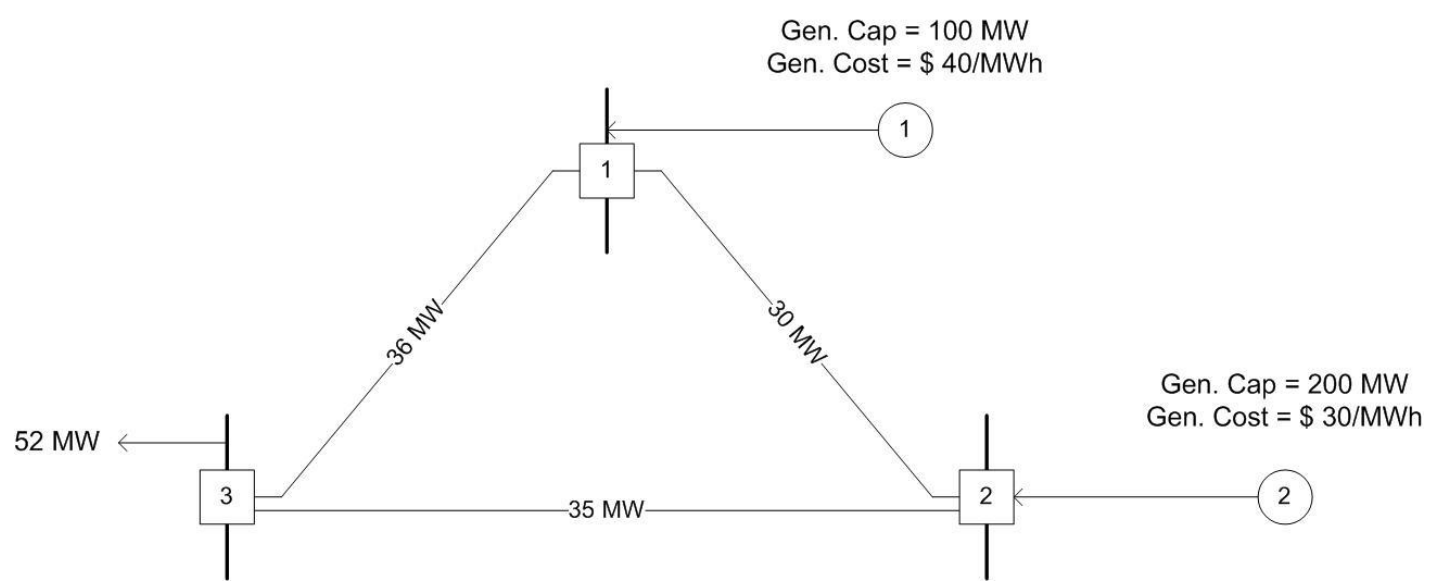

Figure 6. Three-node example

Since there exist two generators in adjacent two nodes, it results in counterflow on the power line connecting node 1 and 2 (For the detail of this issue, please see Appendix showing 
the formation of OPF formulation for the existing network). Thus, in this numerical example, we assess the impact of counterflow on profit and the value of the expansion option.

\section{OPF Problem}

Throughout the numerical example, we solve the OPF problems by using the power flow equations analyzed by Bushnell and Stoft (1995). In Bushnell and Stoft (1995), it is stated that network losses are negligible, voltage support and reactive power are not represented. Thus, linear power equations can be written by using the superposition theorem. This theorem says that net power amount flowing on the lines can be found by considering only one generator in each step. After finding the individual power flows triggered by only one generator, net power flows can be found by adding these individual amounts algebraically. For the detail regarding how to construct OPF problem analyzed by Bushnell and Stoft (1995), please see Appendix.

\section{Demand Lattice}

Since there is one consumption center in the network, it is legitimate to use binomial lattice. We assume that length of period $(\Delta t)$ in binomial lattice is 1 year and modeling horizon is 2 years. In Jin et al. (2011), drift and volatility of demand growth are estimated by analyzing real data from Midcontinent Independent System Operator website. Drift $(\mu)$ and volatility $(\sigma)$ are given as 0.0072 and 0.0094 , respectively. However, in this numerical example, volatility was changed a bit because of maintaining the consistency with other network parameters such as capacity of the power lines. Therefore, we use volatility being equal to 0.13 . We accept that initial demand is $52 \mathrm{MW}$. By using Equations (2) and (3), $u$ and $d$ values are calculated as 1.138 and 0.878. Thus, for demand evolution, binomial lattice illustrated in Figure 7 is created.

$52 \mathrm{MW}$ in demand lattice represents the beginning of the first period and 59.22 (or 45.66 MW) represents the beginning of the second period. We again note that we denote the period 
with $t$ and state with $k . E_{t k}$ denote the demand value at the beginning period $t$ and at state $k$. Thus, $E_{21}=59.22, E_{22}=45.66$ and $E_{11}=52$.

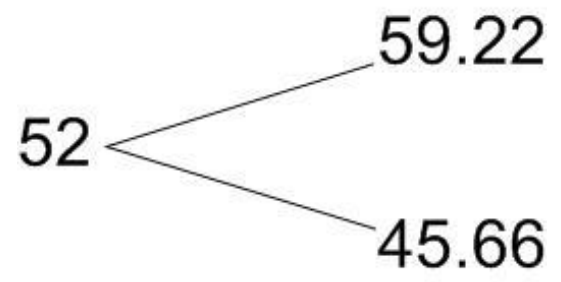

Figure 7. Demand evolution lattice

\section{Investment Valuations}

As can be seen in Figure 6, there are three investment alternatives; between node 1 and 3, between node 1 and 2 and between node 2 and 3. In this section, we create the NPV lattice for the network without investment at first. After that, for each investment alternative, two options (investment made at the beginning of the first year and investment made at the beginning of the second year) are evaluated.

\section{NPV Lattice Without Investment}

Demand lattice triggers the NPV lattice without investment by matching each node of demand lattice to the corresponding node of the NPV lattice except for the terminal nodes. At the end of second year, network is removed and decommissioning cost in incurred. In this example, we assume that decommissioning cost of the existing network is $\$ 250,000$.

We accept that fixed operation and maintenance cost $(c)$ is $\$ 30 /$ hour and risk-free discount rate $(r)$ is 5 percent. Moreover, by using Equation (7) (it is enough to use Equation (7) instead of Equation (8) because we have just two branches in lattices) and $u$ and $d$ values being equal to 1.138 and 0.878 , respectively; we calculate the risk-neutral probability of up movement $(q)$ as 0.66 . Finally, we note that the number of hours in one year $(H)$ is 8760 . 
The result of LMP calculations for the all nodes and the network revenue per hour $\left(N R_{t k}\right)$ is given in Table 2. We note that the units of $E_{t k}$ and $G_{i}$ is MW, the unit of $\pi_{i}$ is $\$ / \mathrm{MWh}$ and the unit of $N R_{t k}$ is $\$ / \mathrm{h}$. Moreover, we remark that $N R_{t k}$ is calculated as the difference between $\pi_{3} E_{t k}$ and $\pi_{1} G_{1}+\pi_{2} G_{2}$ (see Equation (1). We note that we multiply $\pi_{3}$ with $E_{t k}$ because demand is at node 3). For the calculation details regarding LMPs, please see Appendix.

\section{Table 2}

LMP calculation - without investment

\begin{tabular}{ccccccccccc}
\hline$t$ & $k$ & $E_{t k}$ & $\pi_{1}$ & $\pi_{2}$ & $\pi_{3}$ & $G_{1}$ & $G_{2}$ & $\pi_{3} E_{t k}$ & $\pi_{1} G_{1}+\pi_{2} G_{2}$ & $N R_{t k}$ \\
\hline 2 & 1 & 59.22 & 40 & 30 & 50 & 13.44 & 45.78 & 2961 & 1911 & 1050 \\
2 & 2 & 45.66 & 30 & 30 & 30 & 0 & 45.66 & 1369.80 & 1369.80 & 0 \\
1 & 1 & 52 & 30 & 30 & 40 & 0 & 52 & 2080 & 1560 & 520 \\
\hline
\end{tabular}

By using Equation (11), $N P V_{t k}$ (net present value of total profit gained in one year) for the nodes of binomial lattice can be calculated in Table 3. We remark the unit of $N P V_{t k}$ is $\$ /$ year.

Table 3

NPV calculation - without investment

\begin{tabular}{cccc}
\hline$t$ & $k$ & $N R_{t k}$ & $N P V_{t k}$ \\
\hline 2 & 1 & 1050 & $8,509,714$ \\
2 & 2 & 0 & $-250,285$ \\
1 & 1 & 520 & $4,088,000$ \\
\hline
\end{tabular}

For the final lattice, for $t=2$, we have to incur decommissioning cost by adding $(-250,000)(1+0.05)^{-1}$. Thus, for $t=2, k=1$, NPV with decommissioning cost is $\$$ $8,271,619$. For $t=2, k=2$, NPV with decommissioning cost $\$-488,381$. For $t=1, k=1$, in addition to $N P V_{t k}$ value in Table 3, we have to add risk-neutral expected value of the next period. Thus,

$$
4,088,000+(0.66 \cdot 8,271,619-488,381 \cdot 0.34)(1+0.05)^{-1}=9,123,428
$$


Therefore, following lattice without investment can be obtained:

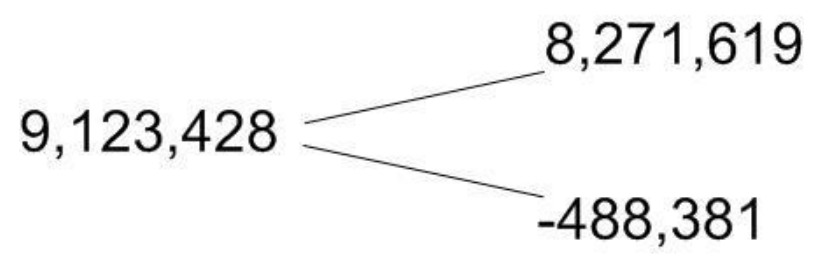

Figure 8. NPV lattice without investment (\$)

\section{NPV Lattice - Investment Between Node 1 and 3}

\section{Option 1 (Investment at the beginning of the first period)}

We assume that a power line is added between node 1 and 3 at the beginning of the first period. We further assume that the capacity of the new line is $4 \mathrm{MW}$ and it has the same susceptance with the existing power line. With this upgrade, fixed operation and maintenance cost increase to $\$ 40 /$ hour. The updated network can be seen in Figure 9. Since a new line is added to the network, underlying OPF problem formulation changes. For the detail of new OPF formulation, please see Appendix.

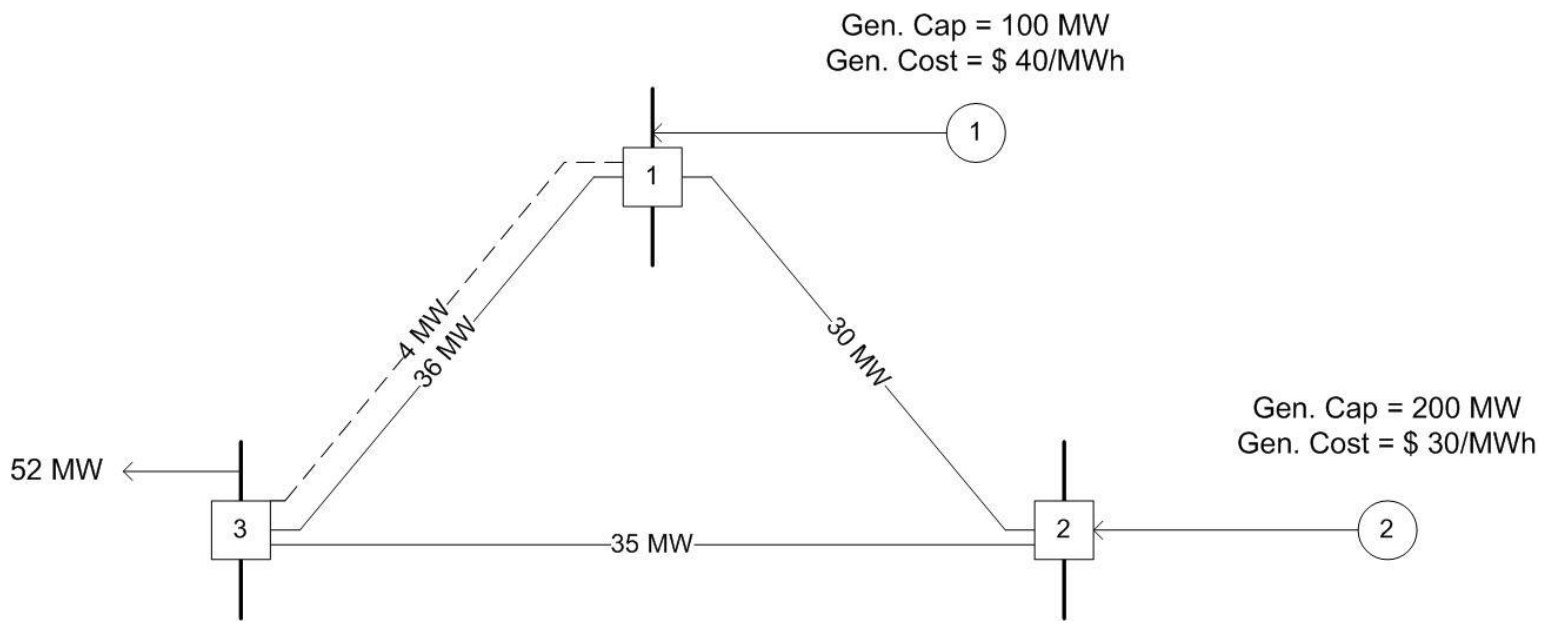

Figure 9. Upgraded network - investment between node 1 and 3 
LMP calculations for the upgraded network are given in Table 4. This table has the same remarks with Table 2 about the unit of values. For the detail regarding the LMP calculations in Table 4, please see Appendix.

\section{Table 4}

LMP calculation - investment between node 1 and 3, option 1

\begin{tabular}{ccccccccccc}
\hline$t$ & $k$ & $E_{t k}$ & $\pi_{1}$ & $\pi_{2}$ & $\pi_{3}$ & $G_{1}$ & $G_{2}$ & $\pi_{3} E_{t k}$ & $\pi_{1} G_{1}+\pi_{2} G_{2}$ & $N R_{t k}$ \\
\hline 2 & 1 & 59.22 & 40 & 30 & 45 & 1.33 & 57.89 & 2664.90 & 1789.90 & 875 \\
2 & 2 & 45.66 & 30 & 30 & 30 & 0 & 45.66 & 1369.80 & 1369.80 & 0 \\
1 & 1 & 52 & 30 & 30 & 30 & 0 & 52 & 1560 & 1560 & 0 \\
\hline
\end{tabular}

By using Equation (11), $N P V_{t k}$ for the nodes of binomial lattice can be calculated as shown in Table 5.

Table 5

NPV calculation - investment between node 1 and 3, option 1

\begin{tabular}{cccc}
\hline$t$ & $k$ & $N R_{t k}$ & $N P V_{t k}$ \\
\hline 2 & 1 & 875 & $6,966,285$ \\
2 & 2 & 0 & $-333,714$ \\
1 & 1 & 0 & $-333,714$ \\
\hline
\end{tabular}

We note that decommissioning cost of the network with a new power line, is assumed to be $\$ 300,000$. We also remark that decommissioning cost of the network with the new line is larger than that of the network before investment.

For the final lattice, for $t=2$, we have to incur decommissioning cost by adding $(-300,000)(1+0.05)^{-1}$. Thus, for $t=2, k=1$, NPV with decommissioning cost is $\$$ $6,680,571$. For $t=2, k=2$, NPV with decommissioning cost $\$-619,429$. For $t=1, k=1$, in addition to $N P V_{t k}$ value, we have to add transmission access charge $(A=\$ 17 M)$ and initial investment cost $(I=\$ 15 M)$ as well as risk-neutral expected value of the next period. Thus,

$$
-333,714+17 M-15 M+(0.66 \cdot 6,680,571-619,429 \cdot 0.34)(1+0.05)^{-1}=5,660,147
$$


Therefore, following lattice with option 1 can be obtained:

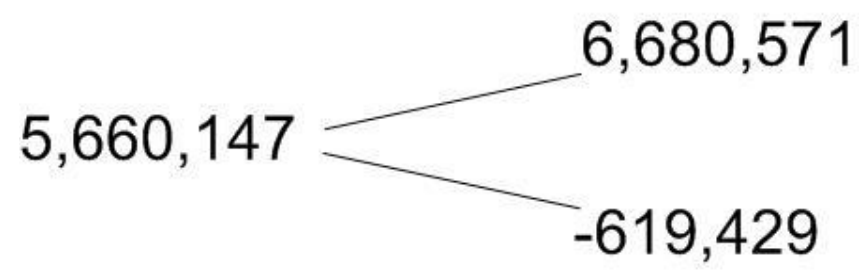

Figure 10. NPV lattice (\$) - investment between node 1 and 3, option 1

Since $\$ 5,660,147$ is less than $\$ 9,123,428$, the value of option 1 is zero for this investment alternative.

\section{Option 2 (Investment at the beginning of the second period)}

We assume that a power line is added between node 1 and 3 at the beginning of the second year. Moreover, we assume that the capacity of this line is $4 \mathrm{MW}$ and it has the same susceptance value.

Table 6 gives the corresponding LMPs and LMP-based revenues. This table has the same remarks with Table 2 about the unit of values. We note here that with demand value being equal to $52 \mathrm{MW}$, LMP calculations are the same 'without investment' situation because there is no investment at that time. For the detail regarding the LMP calculations in Table 6, please see Appendix.

Table 6

LMP calculation - investment between node 1 and 3, option 2

\begin{tabular}{ccccccccccc}
\hline$t$ & $k$ & $E_{t k}$ & $\pi_{1}$ & $\pi_{2}$ & $\pi_{3}$ & $G_{1}$ & $G_{2}$ & $\pi_{3} E_{t k}$ & $\pi_{1} G_{1}+\pi_{2} G_{2}$ & $N R_{t k}$ \\
\hline 2 & 1 & 59.22 & 40 & 30 & 45 & 1.33 & 57.89 & 2664.90 & 1789.90 & 875 \\
2 & 2 & 45.66 & 30 & 30 & 30 & 0 & 45.66 & 1369.80 & 1369.80 & 0 \\
1 & 1 & 52 & 30 & 30 & 40 & 0 & 52 & 2080 & 1560 & 520 \\
\hline
\end{tabular}

By using Equation (11), $\boldsymbol{N} \boldsymbol{P} \boldsymbol{V}_{\boldsymbol{t} \boldsymbol{k}}$ for the nodes of binomial lattice can be calculated as shown in Table 7 (but $\boldsymbol{c}$ is $\$ 40 / \mathrm{h}$ now for the second period). 
Table 7

NPV calculation - investment between node 1 and 3, option 2

\begin{tabular}{cccc}
\hline$t$ & $k$ & $N R_{t k}$ & $N P V_{t k}$ \\
\hline 2 & 1 & 875 & $6,966,285$ \\
2 & 2 & 0 & $-333,714$ \\
1 & 1 & 520 & $4,088,000$ \\
\hline
\end{tabular}

For the final lattice, for $t=2$, we have to incur decommissioning cost by adding $(-300,000)(1+0.05)^{-1}$. Moreover, $A$ and $I$ should be added. Thus, for $t=2, k=1$,

$$
6,966,285+17 M-15 M+(-300,000)(1+0.05)^{-1}=8,680,571
$$

For $t=2, k=2$

$$
-333,714+17 M-15 M+(-300,000)(1+0.05)^{-1}=1,380,571
$$

For $t=1, k=1$, in addition to $N P V_{t k}$ value, we have to add risk-neutral expected value of the next period. Thus,

$$
4,088,000+(0.66 \cdot 8,680,285+0.34 \cdot 1,380,571)(1+0.05)^{-1}=9,986,624
$$

Therefore, following lattice with option 2 can be obtained:

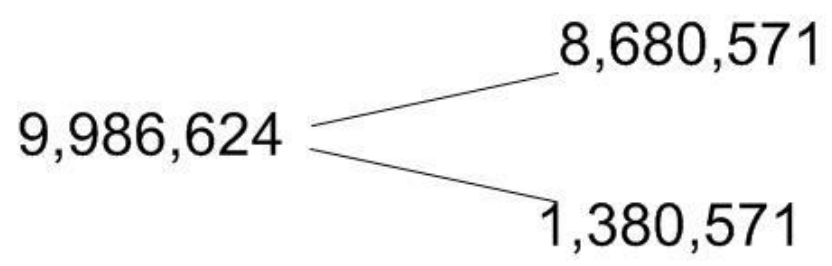

Figure 11. NPV lattice (\$) - investment between node 1 and 3, option 2

Since $\$ 9,986,624$ is larger than $\$ 9,123,428$ ( $\boldsymbol{V}_{\mathbf{1 1}}$ value of NPV lattice without investment), option 2's value is found as the difference between these two values; that is, $\$$ 863,196 . This is the value of investing in the second period. By comparing these two options, it is clear that option 2 turns out to be valuable.

NPV Lattice - Investment Between Node 1 and 2

Option 1 (Investment at the beginning of the first period) 
We consider that an investment is made between node 1 and 2 at the beginning of the first year. For the consistency with the previous investment's parameters, the capacity of the power line is assumed to be $4 \mathrm{MW}$ and the susceptance of the new line is assumed to be equal to that of the current line. Similarly, we consider that operation and maintenance cost is again $\$$ 40/h. The upgraded network can be seen in Figure 12. Moreover, updated power flow equations can be observed in Appendix.

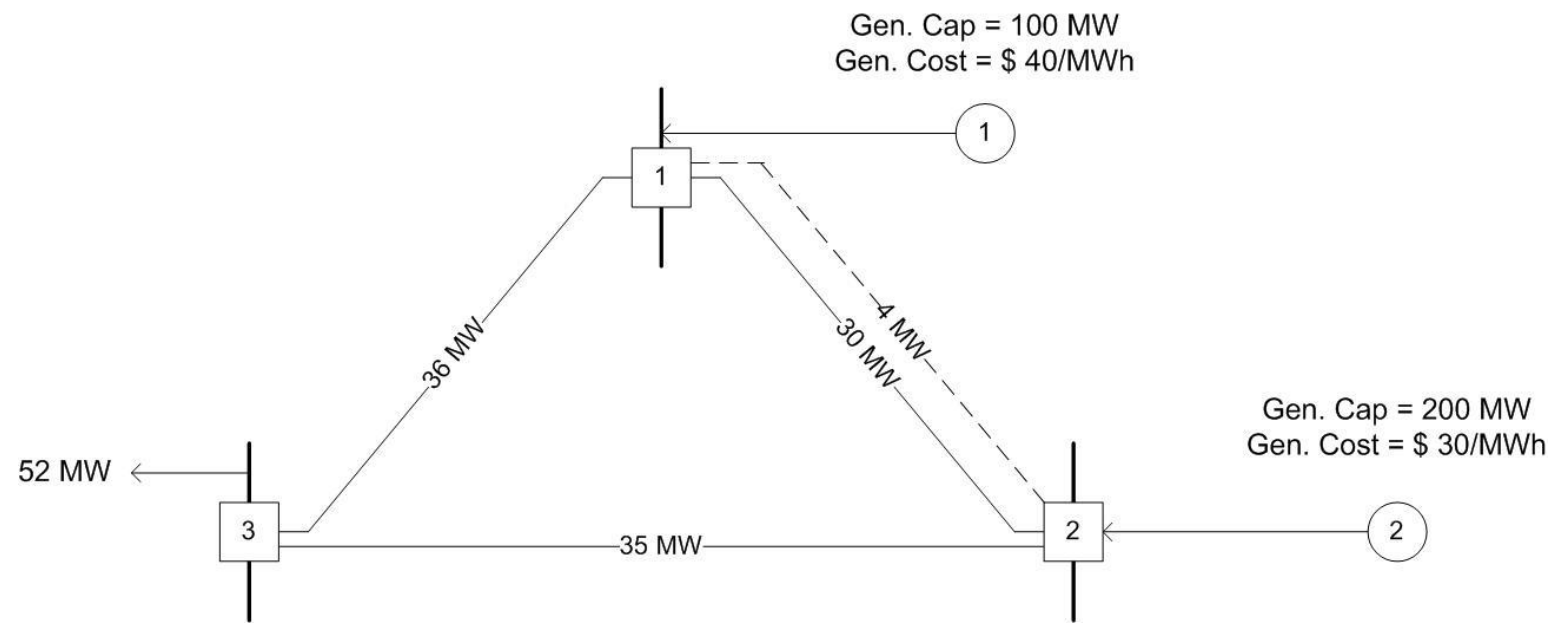

Figure 12. Upgraded network - investment between node 1 and 2

LMP calculations for the upgraded network are given in Table 8. This table has the same remarks with Table 2 about the unit of values. For the detail regarding the LMP calculations in Table 8, please see Appendix.

\section{Table 8}

LMP calculation - investment between 1 and 2, option 1

\begin{tabular}{ccccccccccc}
\hline$t$ & $k$ & $E_{t k}$ & $\pi_{1}$ & $\pi_{2}$ & $\pi_{3}$ & $G_{1}$ & $G_{2}$ & $\pi_{3} E_{t k}$ & $\pi_{1} G_{1}+\pi_{2} G_{2}$ & $N R_{t k}$ \\
\hline 2 & 1 & 59.22 & 40 & 30 & 60 & 2.66 & 56.56 & 3553.20 & 1803.20 & 1750 \\
2 & 2 & 45.66 & 30 & 30 & 30 & 0 & 45.66 & 1369.80 & 1369.80 & 0 \\
1 & 1 & 52 & 30 & 30 & 30 & 0 & 52 & 1560 & 1560 & 0 \\
\hline
\end{tabular}

By using Equation (11), $N P V_{t k}$ for the nodes of binomial lattice can be calculated as 
shown in Table 9.

Table 9

NPV calculation - investment between 1 and 2, option 1

\begin{tabular}{cccc}
\hline$t$ & $k$ & $N R_{t k}$ & $N P V_{t k}$ \\
\hline 2 & 1 & 1750 & $14,266,285$ \\
2 & 2 & 0 & $-333,714$ \\
1 & 1 & 0 & $-333,714$ \\
\hline
\end{tabular}

We note that decommissioning cost of the network is the same as with the previous investment. That is, we accept the same decommissioning cost, which is equal to $\$ 300,000$.

For the final lattice, for $t=2$, we have to incur decommissioning cost by adding $(-300,000)(1+0.05)^{-1}$. Thus, for $t=2, k=1$, NPV with decommissioning cost is $\$$ 13,980,571. For $t=2, k=2$, NPV with decommissioning cost $\$-619,429$. For $t=1, k=1$, in addition to $N P V_{t k}$ value, we have to add transmission access charge $(A=\$ 17 M)$ and initial investment cost $(I=\$ 15 M)$ as well as risk-neutral expected value of the next period. Thus,

$-333,714+17 M-15 M+(0.66 \cdot 13,980,571-619,429 \cdot 0.34)(1+0.05)^{-1}=10,243,941$

Therefore, following lattice with option 1 can be obtained:

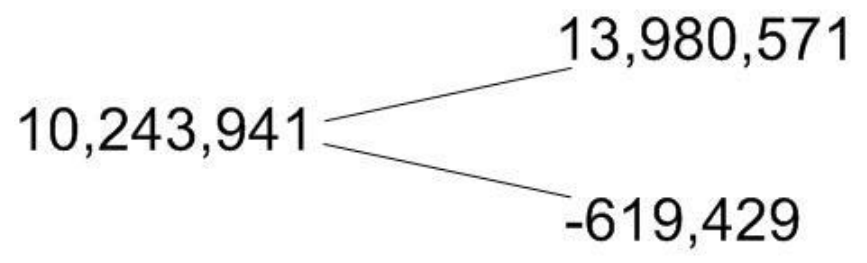

Figure 13. NPV lattice $(\$)$ - investment between node 1 and 2, option 1

Since $\$ 10,243,941$ is larger than $\$ 9,123,428$ ( $V_{11}$ of NPV lattice without investment), the value of the option 1 is found as $\$ 1,120,513$. This is the value of investing between node 1 and 2 at the beginning of the first year.

\section{Option 2 (Investment at the beginning of the second period)}


We assume that a power line is added between node 1 and 2 at the beginning of the second year. Moreover, we assume that the capacity of this line is $4 \mathrm{MW}$ and it has the same susceptance value.

Table 10 gives the corresponding LMPs and LMP-based revenues. This table has the same remarks with Table 2 about the unit of values. We note here that with demand value being equal to $52 \mathrm{MW}$, LMP calculations are the same with 'without investment' situation because there is no investment at that time. For the detail regarding the LMP calculations in Table 10, please see Appendix.

Table 10

LMP calculation - investment between node 1 and 2, option 2

\begin{tabular}{ccccccccccc}
\hline$t$ & $k$ & $E_{t k}$ & $\pi_{1}$ & $\pi_{2}$ & $\pi_{3}$ & $G_{1}$ & $G_{2}$ & $\pi_{3} E_{t k}$ & $\pi_{1} G_{1}+\pi_{2} G_{2}$ & $N R_{t k}$ \\
\hline 2 & 1 & 59.22 & 40 & 30 & 60 & 2.66 & 56.56 & 3553.20 & 1803.20 & 1750 \\
2 & 2 & 45.66 & 30 & 30 & 30 & 0 & 45.66 & 1369.80 & 1369.80 & 0 \\
1 & 1 & 52 & 30 & 30 & 40 & 0 & 52 & 2080 & 1560 & 520 \\
\hline
\end{tabular}

By using Equation (11), $N P V_{t k}$ for the nodes of binomial lattice can be calculated as shown in Table 11 (but $c$ is $\$ 40 /$ h now for the second period).

Table 11

NPV calculation - investment between node 1 and 2, option 2

\begin{tabular}{cccc}
\hline$t$ & $k$ & $N R_{t k}$ & $N P V_{t k}$ \\
\hline 2 & 1 & 1750 & $14,266,285$ \\
2 & 2 & 0 & $-333,714$ \\
1 & 1 & 520 & $4,088,000$ \\
\hline
\end{tabular}

For the final lattice, for $t=2$, we have to incur decommissioning cost by adding $(-300,000)(1+0.05)^{-1}$. Moreover, $A$ and $I$ should be added. Thus, for $t=2, k=1$,

$$
14,266,285+17 M-15 M+(-300,000)(1+0.05)^{-1}=15,980,571
$$


For $t=2, k=2$

$$
-333,714+17 M-15 M+(-300,000)(1+0.05)^{-1}=1,380,571
$$

For $t=1, k=1$, in addition to $N P V_{t k}$ value, we have to add risk-neutral expected value of the next period. Thus,

$$
4,088,000+(0.66 \cdot 15,980,571+0.34 \cdot 1,380,571)(1+0.05)^{-1}=14,570,417
$$

Therefore, following lattice with option 2 can be obtained:

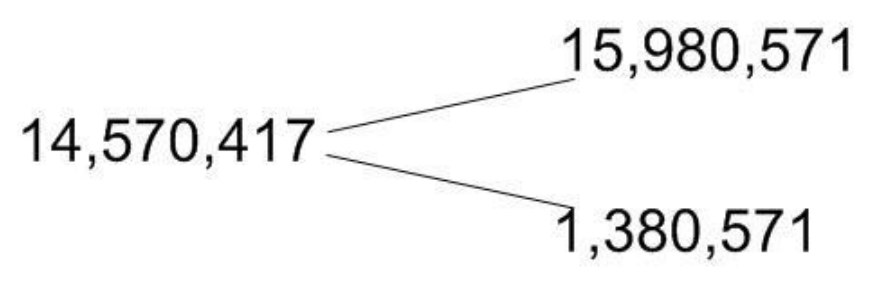

Figure 14. NPV lattice (\$) - investment between node 1 and 2, option 2

Since $\$ 14,570,417$ is larger than $\$ 9,123,428$ ( $V_{11}$ of NPV lattice without investment), the value of option 2 is found as $\$ 5,446,990$. Thus, it can be said that the value of investing between node 1 and 2 at the beginning of the second year is $\$ 5,446,990$. Since the value of the option 2 is larger than that of option 1, option 2 becomes more likely to be implemented.

\section{NPV Lattice - Investment Between Node 2 and 3}

\section{Option 1 (Investment at the beginning of the first period)}

We consider that another power line is added between node 2 and 3. For maintaining the consistency with the previous investment alternatives, the capacity of the new line is assumed to be $4 \mathrm{MW}$ and susceptance of it is equal to that of the existing power line between node 2 and 3 . The upgraded network can be seen in Figure 15. Furthermore, the new power flow formulation can be checked in Appendix. 


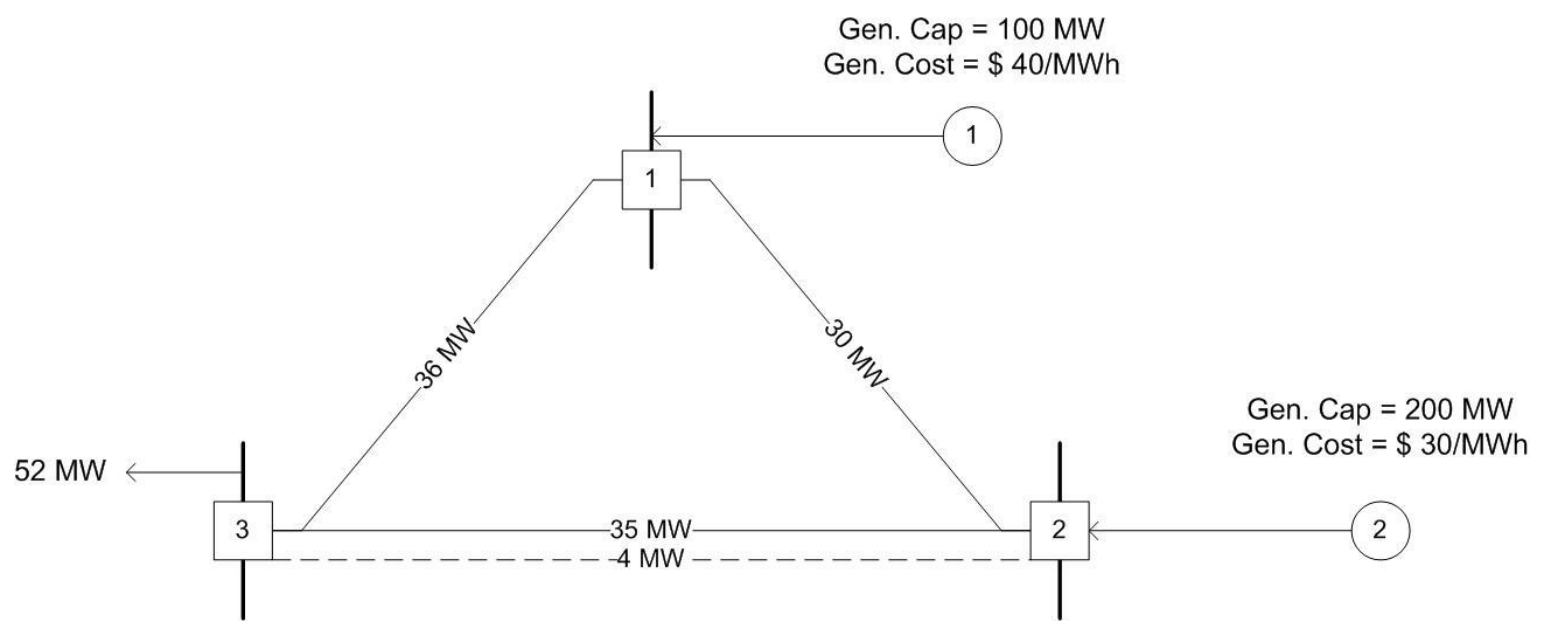

Figure 15. Upgraded network - investment between node 2 and 3

For the network with the corresponding new line, LMP calculations are given in Table 12. This table has the same remarks with Table 2 regarding the unit of values. For the detail of LMP calculations, please see Appendix.

Table 12

LMP calculation - investment between node 2 and 3, option 1

\begin{tabular}{ccccccccccc}
\hline$t$ & $k$ & $E_{t k}$ & $\pi_{1}$ & $\pi_{2}$ & $\pi_{3}$ & $G_{1}$ & $G_{2}$ & $\pi_{3} E_{t k}$ & $\pi_{1} G_{1}+\pi_{2} G_{2}$ & $N R_{t k}$ \\
\hline 2 & 1 & 59.22 & 40 & 30 & 50 & 20.94 & 38.28 & 2961 & 1986 & 975 \\
2 & 2 & 45.66 & 30 & 30 & 30 & 0 & 45.66 & 1369.80 & 1369.80 & 0 \\
1 & 1 & 52 & 40 & 30 & 50 & 6.5 & 45.5 & 2600 & 1625 & 975 \\
\hline
\end{tabular}

By using Equation (11), $N P V_{t k}$ for the nodes of binomial lattice can be calculated as shown in Table 13.

Table 13

NPV calculation - investment between node 2 and 3, option 1

\begin{tabular}{cccc}
\hline$t$ & $k$ & $N R_{t k}$ & $N P V_{t k}$ \\
\hline 2 & 1 & 975 & $7,800,571$ \\
2 & 2 & 0 & $-333,714$ \\
1 & 1 & 975 & $7,800,571$ \\
\hline
\end{tabular}


For the final lattice, for $t=2$, we have to incur decommissioning cost by adding $(-300,000)(1+0.05)^{-1}$. Thus, for $t=2, k=1$, NPV with decommissioning cost is $\$$ $7,514,857$. For $t=2, k=2$, NPV with decommissioning cost $\$-619,429$. For $t=1, k=1$, in addition to $N P V_{t k}$ value, we have to add transmission access charge $(A=\$ 17 M)$ and initial investment cost $(I=\$ 15 M)$ as well as risk-neutral expected value of the next period. Thus,

$$
7,800,571+17 M-15 M+(0.66 \cdot 7,514,857-619,429 \cdot 0.34)(1+0.05)^{-1}=14,318,295
$$

Therefore, following lattice with option 1 can be obtained:

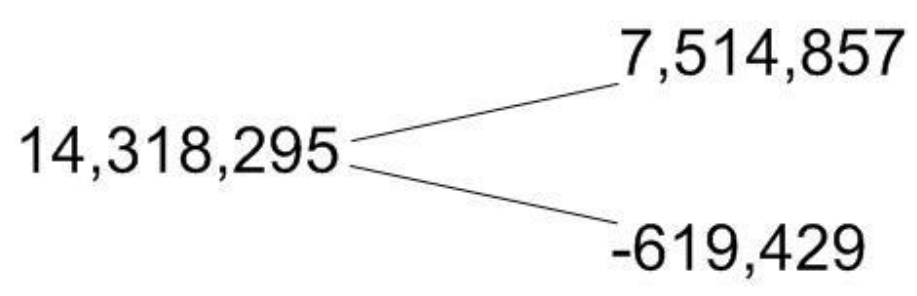

Figure 16. NPV lattice (\$) - investment between node 2 and 3, option 1

Since $\$ 14,318,295$ is larger than $\$ 9,123,428$ ( $V_{11}$ of NPV lattice without investment), option 1's value is found as $\$ 5,194,868$. This is the value of investing between node 2 and 3 at the beginning of the first year.

\section{Option 2 (Investment at the beginning of the second period)}

We assume that a power line is added between node 2 and 3 at the beginning of the second year. Moreover, we assume that the capacity of this line is $4 \mathrm{MW}$ and it has the same susceptance value.

Table 14 gives the corresponding LMPs and LMP-based revenues. This table has the same remarks with Table 2 about the unit of values. We note here that with demand value being equal to $52 \mathrm{MW}$, LMP calculations are the same 'without investment' situation because there is 
no investment at that time. For the detail regarding the LMP calculations in Table 14, please see Appendix.

\section{Table 14}

LMP calculation - investment between node 2 and 3 , option 2

\begin{tabular}{ccccccccccc}
\hline$t$ & $k$ & $E_{t k}$ & $\pi_{1}$ & $\pi_{2}$ & $\pi_{3}$ & $G_{1}$ & $G_{2}$ & $\pi_{3} E_{t k}$ & $\pi_{1} G_{1}+\pi_{2} G_{2}$ & $N R_{t k}$ \\
\hline 2 & 1 & 59.22 & 40 & 30 & 50 & 20.94 & 38.28 & 2961 & 1986 & 975 \\
2 & 2 & 45.66 & 30 & 30 & 30 & 0 & 45.66 & 1369.80 & 1369.80 & 0 \\
1 & 1 & 52 & 30 & 30 & 40 & 0 & 52 & 2080 & 1560 & 520 \\
\hline
\end{tabular}

By using Equation (11), $N P V_{t k}$ for the nodes of binomial lattice can be calculated as shown in Table 15. (but $c$ is $\$ 40 / \mathrm{h}$ now for the second period).

Table 15

NPV calculation - investment between node 2 and 3, option 2

\begin{tabular}{cccc}
\hline$t$ & $k$ & $N R_{t k}$ & $N P V_{t k}$ \\
\hline 2 & 1 & 975 & $7,800,571$ \\
2 & 2 & 0 & $-333,714$ \\
1 & 1 & 520 & $4,088,000$ \\
\hline
\end{tabular}

For the final lattice, for $t=2$, we have to incur decommissioning cost by adding $(-300,000)(1+0.05)^{-1}$. Moreover, $A$ and $I$ should be added. Thus, for $t=2, k=1$,

$$
7,800,571+17 M-15 M+(-300,000)(1+0.05)^{-1}=9,514,857
$$

For $t=2, k=2$

$$
-333,714+17 M-15 M+(-300,000)(1+0.05)^{-1}=1,380,571
$$

For $t=1, k=1$, in addition to $N P V_{t k}$ value, we have to add risk-neutral expected value of the next period. Thus,

$$
4,088,000+(0.66 \cdot 9,514,857+0.34 \cdot 1,380,571)(1+0.05)^{-1}=10,510,486
$$

Therefore, following lattice with option 2 can be obtained: 


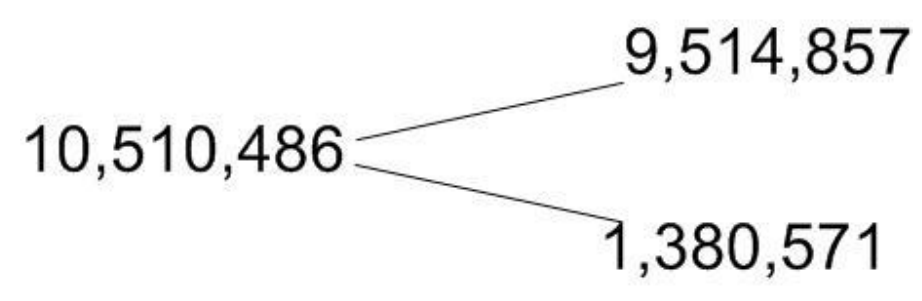

Figure 17. NPV lattice (\$) - investment between node 2 and 3, option 2

Since $\$ 10,510,486$ is larger than $\$ 9,123,428$ ( $V_{11}$ of NPV lattice without investment), value of option 2 is found as $\$ 1,387,058$. This is the value of investing between node 2 and 3 at the beginning of second year. Among two options, first one is more preferable because it has larger value.

After all these calculations, we can present Table 16 as a summary for all investment alternatives.

Table 16

Investment alternatives, their values and times

\begin{tabular}{ccc}
\hline Investment Alternative & Value & Timing of the Investment \\
\hline Node $1-3$ & $\$ 863,196$ & 2 \\
Node $1-2$ & $\$ 5,446,990$ & 2 \\
Node $2-3$ & $\$ 5,194,868$ & 1 \\
\hline
\end{tabular}

The owner has two different flexibilities. One is that he/she can expand the network or not because expansion is not an obligatory issue. The other flexibility is that if the owner decides to invest, he/she can defer the investment, which means he/she can invest at the beginning of any year.

Investment made between node 2 and 3 behaves differently as opposed to other investment alternatives. Similar to the investment made between node 1 and 2 , expansion has 
nonzero value in both periods. However, investing at the beginning of the first year is the most preferable because more revenues are gained. This interesting observation results from the following issue: Since added power line has the same susceptance (thus total susceptance is doubled on that circuit), it dramatically changes the network configuration and more power tries to flow on that circuit. However, since the capacity of the new line is very low (4 MW) with respect to the existing power line $(35 \mathrm{MW})$, the congestion occurs and the degree of congestion becomes more than that of congestion without investment. That results in the advantageous of investing between node 2 and 3 at the beginning of the first year. Therefore, there is no deferring option value. We note that additional observations on the results of Table 16 can be made in a similar fashion.

\section{Conclusion}

In this paper, we developed and analyzed a real options framework that provides the valuation of a transmission owner's option to expand in his/her network. Specifically, under the assumption that the evolution of the demand follows a Geometric Brownian motion process, our framework explicitly accounted for the physical flow of the electric power - economically manifested as locational marginal prices. Through this framework, we showed how the values of the expansion options can be determined in the transmission network. Moreover, given that a specific expansion is already planned, we showed how to value an option to expedite or delay. An extensive numerical example was provided so as to illustrate the key features of our framework with interesting managerial insights.

We note that the framework in this paper can be used as a basis for several expanded studies. For example, additional uncertainties such as fuel costs and regulatory changes can be incorporated. Also, more computationally intense models can be considered where the number of 
periods is into the hundreds (e.g., a 10-year span with a potential decision point in each month). Through such realistic extensions, we hope that this line of study will be helpful in understanding the critical issues in transmission expansion planning faced with substantial and increasing uncertainties in the near future.

\section{References}

Abadie, L. M. and Chamorro, J. M. (2011) Valuing expansions of the electricity transmission network under uncertainty: the binodal case. Energies, 4, 1696-1727.

Blanco, G. A., Pringles, R. M., Olsina, F. G. and Garcés, F. F. (2009) Valuing a flexible regulatory framework for transmission expansion investments, in Proceedings of the PowerTech, Romania, June 28-July 2, 2009.

Blanco, G., Olsina, F., Garcés, F. and Rehtanz, C. (2011) Real option valuation of FACTS investments based on the Least Square Monte Carlo method. IEEE Transactions on Power Systems, 26, 1389-1398.

Bushnell, J. and Stoft, S. (1995) Transmission and generation investment in a competitive electric power industry. Working Paper, University of California Energy Institute, Berkeley, California.

Buygi, M. O., Balzer, G., Shanechi, H. M. and Shahidehpour, M. (2004) Market-based transmission expansion planning. IEEE Transactions on Power Systems, 19, 2060-2067.

California ISO. (2014) Transmission operations ensure the flow of electricity. Available at http://www.caiso.com/market/Pages/TransmissionOperations/Default.aspx ～(accessed December 25, 2014)

California ISO. (2014b) Glossary of terms and acronyms. Available at http://www.caiso.com/Pages/glossary.aspx?View=\{02340A1A-683C-4493-B284- 
8B949002D449\} \&FilterField1=Letter\&FilterValue1=T (accessed December 25, 2014)

California ISO. (2005) Locational marginal pricing (LMP): basics of nodal price calculation. Available at http://www.caiso.com/docs/2004/02/13/200402131607358643.pdf (accessed December 25, 2014).

Cox, J. C., Ross, S. A. and Rubinstein, M. (1979) Option pricing: A simplified approach. Journal of financial Economics, 7, 229-263.

Dixit, A. K. and Pindyck, R. S. (1994) Investment under uncertainty. Princeton University Press, Princeton, NJ.

Garcia, R. C., Contreras, J., Correia, P. F. and Muñoz, J. I. (2010) Transmission assets investment timing using net present value curves. Energy Policy, 38, 598-605.

Hsu, M. (1997) An introduction to the pricing of electric power transmission. Utilities Policy, 6, 257-270.

$\begin{array}{llll}\text { Investopedia. (2014) Discount ate } & \text { Available at }\end{array}$ http://www.investopedia.com/terms/d/discountrate.asp (accessed December 25, 2014).

Jin, S., Ryan, S. M., Watson, J. P. and Woodruff, D. L. (2011) Modeling and solving a largescale generation expansion planning problem under uncertainty. Energy Systems, 2, 209242.

Kirschen, D. S. and Strbac, G. (2004) Fundamentals of power system economics. John Wiley \& Sons, West Sussex, England.

Krause, T. (2003) Evaluation of transmission pricing methods for liberalized markets - a literature survey. Working Paper, Swiss Federal Institute of Technology Zurich, Zurich, Switzerland.

Kuphaldt, T. R. (2006) Lessons in electric circuit volume 1 - DC. Available at 
http://www.ibiblio.org/kuphaldt/electricCircuits/DC/ (accessed December 26, 2014)

Luenberger, D. G. (1997) Investment science. Oxford University Press, New York, New York.

McCalley, J. D. (2007) The DC power flow equations. Available at http://home.eng.iastate.edu/ jdm/ee553/DCPowerFlowEquations.pdf $\quad$ (accessed December 25, 2014)

Mun, J. (2002) Real options analysis - tools and techniques for valuing strategic investments and decisions. John Wiley \& Sons, Inc, Hoboken, New Jersey.

Osthues, M., Rehtanz, C., Blanco, G. and Negnevitsky, M. (2014) Real options on power system planning under uncertainties and efficiency constraints, in Proceedings of the 18th Power Sytems Computation Conference, Poland, August 18-22, 2014.

Pe'rez-Arriaga, I. J., Olmos, L. and Rivier, M. (2013) Transmission Pricing. In: J. Rosello'n and T. Kristiansen, eds. Financial Transmission Rights: Analysis, Experiences and Prospects. Springer-Verlag, London, England.

Pringles, R., Olsina, F. and Garces, F. (2014) Designing regulatory frameworks for merchant transmission investments by real options analysis. Energy Policy, 67, 272-280.

Vasquez, P. and Olsina, F. (2007) Valuing flexibility of DG investments in transmission expansion planning, in Proceedings of the Power Tech 2007, Lausanne, July 1-5, 2007.

Wang, C. H. and Min, K. J. (2006) Electric power generation planning for interrelated projects: a real options approach. IEEE Transactions on Engineering Management, 53, 312-322.

Zacks. (2014) Education: value investing. Available at http://www.zacks.com/education/articles.php?id=22 (accessed December 25, 2014).

\section{Appendix: Derivation of LMP Differences OPF Problem}


For the detail of OPF problem, please see the relevant references Kirschen and Strbac (2004) and McCalley (2007).

\section{Approximation of AC Power Flow Equations}

For the detail regarding the approximation procedure, please see the relevant references Kirschen and Strbac (2004) and McCalley (2007).

\section{OPF Formulation for the Network Without Investment}

As stated in the main text, it is assumed that network losses are negligible, voltage drops and reactive powers are not represented (see e.g., Bushnell and Stoft (1995), Kirschen and Strbac (2004)). Thus, we are allowed to use the linear power flow equations found by dividing the power dispatched from one generator with respect to the path's total susceptance (Bushnell and Stoft (1995) explains this principle by using admittance. However, admittance and susceptance are equivalent in this context because we are just worried about the easiness of flow on the power lines. Therefore, it does not matter to use admittance or susceptance in this context). This principle is also known as power transfer distribution factors. Therefore, superposition principle can be utilized. In this principle, only one generator is taken into for each step and power amounts on the lines are found. At the end, power amounts on the lines are summed algebraically and net power amounts are obtained.

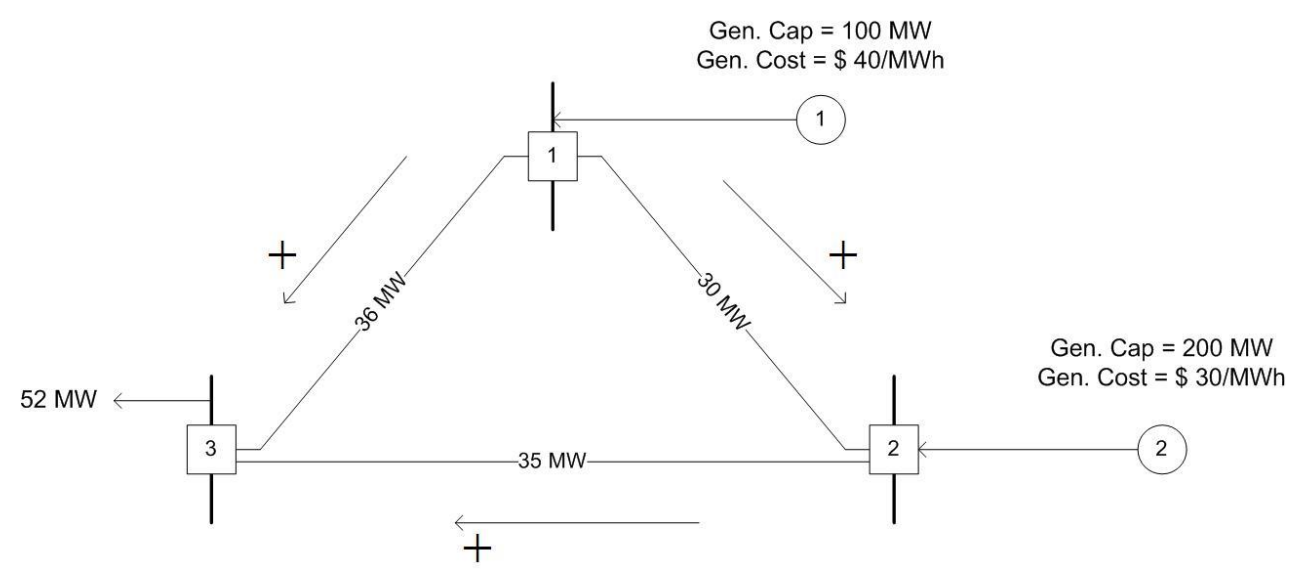


In this example, we assume that the direction of power flows occurs as seen in above figure. Let's consider the generator 1, at first. If power is dispatched from this generator, then it flows in two paths: node 1 to 3 and node 1 to 2 to 3 . If two power lines are connected serial, then total susceptance is found by

$$
\frac{1}{b_{123}}=\frac{1}{b_{12}}+\frac{1}{b_{23}}=\frac{1}{b}+\frac{1}{b}=\frac{2}{b} \Rightarrow b_{123}=\frac{b}{2}
$$

where $b_{123}$ denotes the total susceptance of path from node 1 to 2 to $3, b_{12}$ denotes the susceptance of power line connecting node 1 and 2 and $b_{23}$ denotes the susceptance of power line connecting node 2 and 3. Here, we denote one unit susceptance as $b$. Let $V_{1}$ and $V_{3}$ denote the voltage at node 1 and 3, respectively. By using the Ohm's law, we can write that

$$
V_{1}-V_{3}=\frac{L_{13}}{b_{13}}=\frac{L_{123}}{b_{123}}=\frac{L_{13}}{b}=\frac{L_{123}}{\frac{b}{2}}
$$

where $L_{13}$ denotes the power flow from node 1 to 3 and $L_{123}$ denotes the power flow from node 1 to 2 to 3 . Therefore, from the above equation, we can say that $2 L_{123}=L_{13}$. Hence, following power flow equations can be written:

$$
L_{12}=\frac{1}{3} G_{1}, \quad L_{13}=\frac{2}{3} G_{1}, \quad L_{23}=\frac{1}{3} G_{1}
$$

where $G_{i}$ denotes the dispatch amount of generator at node $i$. Let's now consider the second generator. If power is dispatched from the generator, then it flows in two paths: from node 2 to 1 to 3 and from node 2 to 3 . By using the Ohm's law, we can write that

$$
V_{2}-V_{3}=\frac{L_{23}}{b_{23}}=\frac{L_{213}}{b_{213}}=\frac{L_{23}}{b}=\frac{L_{213}}{\frac{b}{2}} \Rightarrow L_{23}=2 L_{213}
$$

Hence, power flows equations can be written in the following form: 


$$
L_{12}=-\frac{1}{3} G_{2}, \quad L_{13}=\frac{1}{3} G_{2}, \quad L_{23}=\frac{2}{3} G_{2}
$$

By summing up these power flows, one can reach the net power amounts as follows:

$$
\begin{aligned}
L_{12} & =\frac{1}{3} G_{1}-\frac{1}{3} G_{2} \\
L_{13} & =\frac{2}{3} G_{1}+\frac{1}{3} G_{2} \\
L_{23} & =\frac{1}{3} G_{1}+\frac{2}{3} G_{2}
\end{aligned}
$$

We note that net power flows on the line connecting node 1 and 2 have reverse direction. Thus, the sign of these power flows are reverse. In fact, the power flows on the line connecting node 1 and 2 do not cancel each other. Rather, superposition principle is used for finding the actual power flow on the line. Thus, we can call the individual power amounts triggered by each generator as fictitious (see e.g., Kuphaldt (2006)).

Thermal limit constraints of the power lines and capacity constraints for the generator are added.

$$
\begin{gathered}
-30 \leq L_{12} \leq 30 \\
-36 \leq L_{13} \leq 36 \\
-35 \leq L_{23} \leq 35 \\
0 \leq G_{1} \leq 100 \\
0 \leq G_{2} \leq 200
\end{gathered}
$$

Since we do not know right direction of power flows on the lines, we add the capacity of the power lines with both negative and positive signs. Finally, the demand amount should be equal to the total amount of power dispatched. Thus, as a final equation, we add the following constraint: 


$$
G_{1}+G_{2}=52
$$

The objective of the OPF problem is to minimize the generation costs. Thus, the objective function is

$$
\min _{G_{1}, G_{2}} 40 G_{1}+30 G_{2}
$$

\section{LMP and LMP-based Revenue Calculation Without Investment}

We remind that a demand value is denoted by $E_{t k}$ at the beginning of period $t$ and state $k$.

$$
E_{21}=59.22
$$

Solution of OPF: We consider at first the cheapest generator (generator 2). If all 59.22 MW is dispatched from this generator, then $L_{23}=39.48 \mathrm{MW}, L_{12}=-19.74 \mathrm{MW}$ and $L_{13}=$ 19.74 MW. However, $L_{23}>\overline{L_{23}}$. Thus, we have to increase the dispatch amount of generator 1 and simultaneously decrease the dispatch amount of generator 2. Let $\Delta G_{1}$ and $\Delta G_{2}$ be the change in dispatch of generators 1 and 2 , respectively. Thus, it should be $\Delta G_{1}+\Delta G_{2}=0$ and $\frac{1}{3} \Delta G_{1}+$ $\frac{2}{3}\left(59.22+\Delta G_{2}\right)=35$. The solution of this set of equations are $\Delta G_{1}=13.44$ and $\Delta G_{2}=$ -13.44. Thus, $G_{1}=13.44 M W$ and $G_{2}=45.78 M W$. Since the power flows on the other lines resulting from the dispatch do not violate the capacity limits, we can say that this is the optimal solution.

LMP at node 1: In order to calculate LMP at node 1, we increase the load amount at this node. After that, we at first check the cheapest generator (generator 2) to supply this additional load. If the dispatch amount of this generator is increased by $1 \mathrm{MW}$, then $\frac{2}{3} \mathrm{MW}$ power flows from node 2 to node 1 . In this case, $L_{23}=35.33 \mathrm{MW}$ which violates $\overline{L_{23}}$. Thus, we check the second cheapest generator in order to supply $1 \mathrm{MW}$ additional load. Since remaining capacity of this generator is sufficient for supplying, then this generator is dispatched. The change in total 
system cost is $\$ 40 / \mathrm{h}$, and thus, LMP at node 1 is $\$ 40 / \mathrm{MWh}$.

LMP at node 2: We increase load amount at node 2. At first, the cheapest generator should be checked to supply $1 \mathrm{MW}$ load. Since the remaining capacity of this generator is sufficient for supplying, then this generator is dispatched. The change in total system cost is $\$$ 30/h and LMP at this node is \$ 30/MWh.

LMP at node 3: The load amount at this node is increased by $1 \mathrm{MW}$. We check the cheapest generator at first. It is observed that if $1 \mathrm{MW}$ load is supplied by this generator, then $L_{23}=35.66$. Since $L_{23}>\overline{L_{23}}$, it means that we cannot dispatch the generator 2 on its own. Secondly, we have to check the first generator to supply $1 \mathrm{MW}$ load. If the dispatch of this generator is increased by $1 \mathrm{MW}$, then $L_{23}=35.33$, which also violates $\overline{L_{23}}$. Then, it means that we cannot dispatch this generator by its own. At this point, we find a combinational dispatch of the generators. Let $\Delta G_{1}$ and $\Delta G_{2}$ be the changes in dispatch of the generators 1 and 2 , respectively. Then,

$$
\begin{gathered}
\Delta G_{1}+\Delta G_{2}=1 \\
\frac{1}{3} \Delta G_{1}+\frac{2}{3} \Delta G_{2}=0
\end{gathered}
$$

where first equation represents that change in total dispatch should be equal to one additional demand and second equation represents that power flow on the line connecting node 2 and 3 must stay at $35 \mathrm{MW}$. If we solve this set of equations, we get $\Delta G_{1}=2$ and $\Delta G_{2}=-1$. Thus, the change in total system cost is $2 M W \cdot \$ 40 / M W h-1 M W \cdot \$ 30 / M W h=\$ 50 / h$ and LMP at this node is $\$ 50 / \mathrm{MWh}$.

Network Revenue: In summary, at the end of all these calculations, the following values are obtained regarding LMPs at each node, demand value and the dispatch amount of the generators: $\pi_{1}=40, \pi_{2}=30, \pi_{3}=50, D_{3}=59.22, G_{1}=13.44$ and $G_{2}=45.78$. By using 
Equation (1) and these values, network revenue denoted by $N R_{21}$ in the main text are calculated as $\$ 1050 / \mathrm{h}$.

$$
E_{22}=45.66
$$

Solution of OPF: We consider at first the cheapest generator (generator 2). If all 45.66 MW is dispatched from this generator, then $L_{23}=30.44 M W, L_{12}=-15.22 M W$ and $L_{13}=$ 15.22 MW. Since none of these power flows violates the capacity limits of the corresponding power lines, this is accepted as optimal solution. Thus, at optimality, $G_{1}=0 M W$ and $G_{2}=$ 45.66 MW.

LMP at node 1: We increase the load amount at node 1. After that, we at first check the cheapest generator to supply this additional load. If the dispatch amount of this generator is increased by $1 \mathrm{MW}$, then $\frac{1}{3} \mathrm{MW}$ flows from node 2 to 3 to 1 . Additionally, $\frac{2}{3} \mathrm{MW}$ power flows from node 2 to node 1 directly. In this case, $L_{12}=-15.89, L_{13}=14.89$ and $L_{23}=30.77$. Since none of these values violates the capacity limits of the corresponding power lines, generator 2 can be dispatched to supply the additional load at node 1 . Thus, the change in total system cost is $\$ 30 / \mathrm{h}$, and thus, LMP at node 1 is $\$ 30 / \mathrm{MWh}$.

LMP at node 2: We increase load amount at node 2. At first, the cheapest generator should be checked to supply $1 \mathrm{MW}$ load. Since the remaining capacity of this generator is sufficient for supplying, then this generator is dispatched. The change in total system cost is $\$$ 30/h and LMP at this node is \$ 30/MWh.

LMP at node 3: The load amount at this node is increased by $1 \mathrm{MW}$. We check the cheapest generator at first. It is observed that if $1 \mathrm{MW}$ load is supplied by this generator, then $L_{23}=31.11 \mathrm{MW}, L_{12}=-15.55 \mathrm{MW}$ and $L_{13}=15.55$. Since none of these violates the capacity limits of the corresponding power lines, generator 2 can be dispatched to supply the 
additional load at node 3 . Thus, the change in total system cost is $\$ 30 / \mathrm{h}$ and LMP at this node is \$30/MWh.

Network Revenue: In summary, at the end of all these calculations, the following values are obtained regarding LMPs at each node, demand value and the dispatch amount of the generators: $\pi_{1}=30, \pi_{2}=30, \pi_{3}=30, D_{3}=45.66, G_{1}=0$ and $G_{2}=45.66$. By using Equation (1) and these values, network revenue denoted by $N R_{22}$ in the main text are calculated as $\$ 0 / \mathrm{h}$.

$$
E_{11}=52
$$

Solution of OPF: We consider at first the cheapest generator. If all $52 \mathrm{MW}$ is dispatched from this generator, then $L_{23}=34.67 \mathrm{MW}, L_{12}=-17.33 \mathrm{MW}$ and $L_{13}=17.33 \mathrm{MW}$. Since none of the power flows violates the capacity limits of the corresponding power lines, this is accepted as optimal solution. Thus, at optimality, $G_{1}=0 \mathrm{MW}$ and $G_{2}=52 \mathrm{MW}$.

LMP at node 1: We increase the load amount at node 1. After that, we at first check the cheapest generator to supply this additional load. If the dispatch amount of this generator is increased by $1 \mathrm{MW}$, then $\frac{1}{3} \mathrm{MW}$ flows from node 2 to 3 to 1 . Additionally, $\frac{2}{3} \mathrm{MW}$ power flows from node 2 to node 1 directly. In this case, $L_{12}=-18 \mathrm{MW}, L_{13}=17 \mathrm{MW}$ and $L_{23}=35 \mathrm{MW}$. Since none of these values violates the capacity limits of the corresponding power lines, generator 2 can be dispatched to supply the additional load at node 1 . Thus, the change in total system cost is $\$ 30 / \mathrm{h}$, and thus, LMP at node 1 is $\$ 30 / \mathrm{MWh}$.

LMP at node 2: We increase load amount at node 2. At first, the cheapest generator should be checked to supply $1 \mathrm{MW}$ load. Since the remaining capacity of this generator is sufficient for supplying, then this generator is dispatched. The change in total system cost is $\$$ 30/h and LMP at this node is \$ 30/MWh. 
LMP at node 3: The load amount at this node is increased by $1 \mathrm{MW}$. We check the cheapest generator at first (generator 2). It is observed that if $1 \mathrm{MW}$ load is supplied by this generator, then $\frac{2}{3}$ MW additional power flows on the line from node 2 to 3 . Thus, $L_{23}=$ 35.33 MW. Since $L_{23}>\overline{L_{23}}$, it means that we cannot dispatch the generator 2 on its own. Secondly, we have to check the first generator to supply $1 \mathrm{MW}$ load. If the dispatch of this generator is increased by $1 \mathrm{MW}$, then $L_{23}=35 \mathrm{MW} \leq \overline{L_{23}}$. Thus, additional load at node $3 \mathrm{can}$ be supplied from generator 1 . The change in total system cost is $\$ 40 / \mathrm{h}$ and LMP at this node is $\$$ 40/MWh.

Network Revenue: In summary, at the end of all these calculations, the following values are obtained regarding LMPs at each node, demand value and the dispatch amount of the generators: $\pi_{1}=30, \pi_{2}=30, \pi_{3}=40, D_{3}=52, G_{1}=0$ and $G_{2}=52$. By using Equation (1) and these values, network revenue denoted by $N R_{11}$ in the main text are calculated as $\$ 520 / \mathrm{h}$.

For the upgraded network with investment, same procedure can be followed for calculating the LMPs and LMP-based revenues.

\section{OPF Formulation for the Network With Investment Between Node 1 and 3}

The same procedure with 'without investment' case can be followed. However, the susceptance of the power line between node 1 and 3 is now doubled.

\section{OPF Formulation for the Network With Investment Between Node 1 and 2}

The same procedure with 'without investment' case can be followed. However, the susceptance of the power line between node 1 and 2 is now doubled.

\section{OPF Formulation for the Network With Investment Between Node 2 and 3}

The same procedure with 'without investment' case can be followed. However, the susceptance of the power line between node 2 and 3 is now doubled. 